\title{
Personal compatibility of physician and patient
}

\author{
E.V. Mamonova ${ }^{1 *}, E . V$. Sidorina $^{2}$, and $O . V$. Suvorova $^{3}$ \\ ${ }^{1}$ Nizhny Novgorod state pedagogical University K. Minin, Nizhny Novgorod, Russia \\ ${ }^{2}$ Nizhny Novgorod state pedagogical University K. Minin, Nizhny Novgorod, Russia \\ ${ }^{3}$ Nizhny Novgorod state pedagogical University K. Minin, Nizhny Novgorod, Russia
}

\begin{abstract}
The article discusses and details the interpersonal compatibility of the physician and the patient in the course of their interaction, which is the basis of medicine, the platform on which the treatment process is based. An analysis of the concept of compatibility has been carried out, which is presented in domestic and foreign psychological literature. The analysis suggests that personal compatibility is a function of satisfaction with collaborative action, since it is initially necessary to interact, followed by compatibility, the result of which is rebuilt in satisfaction. By analyzing the statements of domestic and foreign psychologists, compatibility can be imagined in the form of an ideal combination of qualities and senses, resulting in the maximum result in carrying out various actions. Thus, personal compatibility is important in all types of social relationships, but it plays a special role in the long-term relationship between the doctor and the patient. The study concluded that the compatibility of the physician and the patient, as well as the building of an informed relationship between them, is significantly influenced by the presence of similar personal characteristics. The similarity of the personal characteristics of the doctor and the patient allows achieving the best result in their joint activity.
\end{abstract}

\section{A problem statement}

A modern psychologist - a specialist working in a dynamic, developing environment, providing assistance in various fields, including health care. According to modern research, the practicing psychologist has such an important characteristic as tolerance to uncertainty, which is especially needed in our reality [1]. With a relevant approach to health, a person visits a doctor on a regular basis and chooses a specialist for further treatment with whom it will comfortably cooperate for a long time. In such cases, the question of psychological compatibility is acute.

The problem of psychological compatibility is complex and not well researched in psychology, as it lacks a complete and complete theory of compatibility, which in turn affects all levels of the study, from terminology to the development of interoperability models in different activities. The term compatibility is synonymous with psychological compatibility, cohesion, a community of interests, coherence, non-conflict.

\footnotetext{
*Corresponding author: prof-ped.gpa@mail.ru
} 
One of the first founders of the classification of personal compatibility is M. Shaw. In 1971, in his classification, he identified types of personal compatibility.

Compatibility is mainly related to the realm of interpersonal relationships, whether between individuals or within groups. The interpersonal compatibility of people with each other, i.e. in the diaspora, manifests itself in the mutual acceptance of partners in communication and joint activities and is based on the similarity of principles, social foundations, interests, the optimal combination of features of psychological processes, and characteristics [2].

The study of interpersonal compatibility between doctor and patient in the course of their interaction is relevant because the relationship between doctor and patient is the basis of medicine, the platform on which the process of treatment is based. The doctor-patient relationship has a significant impact on the course and success of treatment. Since a doctor cannot refuse an unsuitable patient, he must be able to build a relationship with him that does not adversely affect the treatment process $[3,4,5]$.

One generally accepted definition of compatibility cannot be found. In a thorough analysis of the concepts of compatibility, we take more detailed definitions and interpretations. The term compatibility in psychological dictionaries is interpreted as follows:

1) The compatibility of people in a particular group - a social characteristic of a group by psychological parameters, which is reflected in the ability of the members of that group to coordinate their activities and to rationalize relations among themselves in any common collective action [6];

2) People's compatibility with each other - mutual acceptance of partners in communication and collective action, originating in a similarity of principles, social attitudes, interests, motives, needs, a peculiarity for both psychological and physiological reflexes, and other important personality features vital for interaction between individuals [7].

Based on the statements of K.K. Platonov, compatibility is defined in its psychological essence as interacting by following a certain pattern of behavior, as expressed by its characteristic actions.

The process of compatibility in the introduction of mental and social processes is very complicated, as compatibility can be not only favorable or negative, but it's also possible to manifest all various types and degrees [8].

On the basis of the statements of the Doctor of Psychological Sciences A.L. Svetzicki, compatibility according to its psychological essence should induce individual subjects of the group to concerted activity, which is based on their ideal connection or combination [9].

Based on the statements of V.V. Boyko, A.G. Kovalev, and V.N. Panferov, compatibility is characterized by such a common set of qualities of each individual member of the group, when their efficiency reaches the maximum value [10].

Based on the judgments of Russian psychology, the concept of compatibility is interpreted on the basis of the characteristics of the interaction of several subjects, which determine the maximum permissible result under certain conditions with minimal energy consumption for participants of a given group. Compatibility determines the basis for the sympathy, conformance, and association of the psychological and physiological characteristics of each individual [11].

In foreign psychology, approaches that explain compatibility are divided into needs and behavioral. As this separation shows, compatibility depends on the degree to which needs are met together, as well as the cohesion of each individual of a particular group.

American psychologist M. Shaw distinguishes two types of interpersonal compatibility: 
- The required compatibility - shows that in some cases it is based on similarities in the required performance of the group members, in other cases, it is a question of complementarity of the characteristics, or of some complex interactions;

- behavioral compatibility - shows that certain personal characteristics of group members determine the rank-and-file behavioral patterns capable of producing compatibility, or show that compatibility is not possible [12].

The type of interaction between group members significantly affects the individual compatibility elements. For example, colleagues who are sufficiently compatible with respect to their profession in many ways may in fact have no hint of compatibility in offthe-job interaction, Since this compatibility is characterized by a direct relationship between goals in life and the set of interests of individuals.

\subsection{The objective of the work}

The theory of interpersonal relationships B. Schutz is a combination of hypotheses that can explain the relationships between individuals. The main objective of this theory is the idea that each individual has a specific character of social orientation in relation to other people, which reveals their attitudes and behavior in the interaction between individuals.

Define acceptable types of compatibility between people on the basis of V. Schutz. When designating all types, two behaviors are acceptable: in the first variant, how the person shows himself and manifests himself to the people around him; The second is the behavior that people around you want to see.

Based on the statements of M. Shaw and V. Schutz, it can be concluded that personal compatibility is an important part of a person's quality of social life, as a person will strive to meet his or her needs, thus he cannot avoid contact with others [13].

Based on the models of mental interaction of V.N. Panferov, the concept of compatibility has a psychological-social basis, which is based on one type of interpersonal interaction, which in turn characterizes its scientific base. We will analyze the attributes of the concept of compatibility on the basis of the following definitions and try to compare them.

Compatibility and all related justifications may be divided into the following groups:

1) On cognitive-behavioral factors (interaction, organization, consistency of behavior, ability to act together, a simultaneous manifestation of psychomotor functions);

2) Emotional type (interpersonal harmony, empathy, satisfaction with the course and outcome of joint action);

3) On a systemic type (relationships between individuals, similarities between them, relationships between group members);

4) By the degree of human interaction (indivisibility, common unity, indivisibility).

The concept of compatibility can be understood as a dependency of feelings and emotions that can be both positive and negative. In analyzing the above definitions, we can point out that personal compatibility is a function of satisfaction with joint action because it is necessary first of all to have interaction, then to have compatibility, the result of which is reborn to satisfaction. By analyzing the statements of domestic and foreign psychologists, compatibility can be imagined in the form of an ideal combination of qualities and senses, resulting in the maximum result in carrying out various actions. Accordingly, when the needs of people are reciprocated through their joint actions, they must be seen as compatible. Compatibility between individuals begins on the basis of similarity and complementarity, but if the conditions are not met, there will be no compatibility between individuals [13].

The indicator of overall interaction and formation of full relationships between individuals is characterized by social, psychological, and psycho-physiological 
compatibility. These factors can manifest themselves in all kinds of human interaction. The necessity of any of the factors is characterised by the type of activity performed, the objectives, the basis, and different requirements [14].

At the social level, the indicators of compatibility are people's level of education, attitude to interaction, activity, attitude to joint activity, co-orientation of individuals, meaningful orientation, matching of needs, etc.

Indicators of compatibility at the psychological level primarily determine the similarity and comparison of people on various mental indicators and qualities such as intelligence, attention, anxiety, communication, temperament, etc. This type of compatibility shows how likely it is that people will interact to perform joint activities [15].

Thus, personal compatibility is important in all types of social relationships, but it plays a special role in the long-term relationship between the doctor and the patient.

One of the most important conditions for the establishment of a doctor-patient relationship is the feeling of support. If the patient realizes that the doctor is willing and able to help rather than harm, the patient will be much more involved in the treatment process. When the doctor shows understanding, the patient is sure that his complaints are heard, recorded in the mind of the doctor, and the doctor thinks about them. This feeling is strengthened when the doctor says: «I hear you and understand you» - or expresses it with eye contact or a nod of the head. Respect implies recognition of the value of the individual as an individual. We also tend to believe that compassion is the key to establishing the doctor's cooperation with the patient, as we need to be able to put ourselves in the position of the patient and look at the world through his eyes [16].

In considering the first premise of the relationship, one can distinguish that the basis is the type and type of information, a number of indicators, and certain criteria that the patient learns and collects when visiting a doctor, which he chooses and with which he will need to interact and communicate. If it is possible to choose a physician, the patient collects the necessary information about the physician, asks the patients who have been or are still being treated by the physician, listens to the various comments of the staff of the medical institution, with whom this doctor works and contacts [17], [18].

One of the first people to pay attention to the fundamentals and indicators that shape the relationship between the doctor and the patient was 3 . The bases are formed on the basis of two mechanisms, which in any case arising from any interaction between the doctor and the patient.

The first is the transfer mechanism, which is formed by the experience in the patient of past experience of interaction with people that are of special importance, and the projection of this relationship on the doctor. Based on the results of the study Z. Freud, a physician at a special subconscious level projects himself as a person emotionally important to the patient in childhood [19].

A more detailed examination of the mechanism makes it possible to emphasize that the attitude of the patient to the doctor is directly dependent on the relationship with other doctors, the attitude of the individual doctor to the patient, memories from a childhood of the first experience with doctors. Accordingly, if the patient has seen kindness, empathy and has experienced a level of professional training, and is satisfied by all criteria, this attitude will be formed as positive. With indifference to the patient, inappropriate behavior, unprofessional diagnosis, and prescription of treatment, as with the general level of treatment, a negative and distrustful attitude towards all doctors will develop.

The second is the mechanism of counter transference, which is based on the physician's past experience of interacting with people, colleagues, children who are of special importance, and projecting this relationship on the patient. If the patient has an association of a doctor with a more experienced adult who will put him out of his misery, the doctor, on the contrary, associates the patient in this case with a dependent child who needs care, 
understanding, and helps. Based on the development of previous interactions with patients, the doctor projects positive (positive) or negative (negative) attitudes on the patient.

A more detailed examination of this mechanism reveals that a doctor may have stereotypes about certain types of patients as well as different categories such as age, gender, and others that may form a relationship indicator.

Similarly, from the above, it can be stated that the most appropriate result of combining these mechanisms would be one in which both positive transference and positive counter transference are present. In this case, the relationship between the patient and the doctor will be one that is based on trust, respect, understanding of each other, and cooperation in common interaction. In a different outcome, when negative transference and negative counter transference prevail, the physician and the patient need to understand the cause of this inappropriate and negative relationship, Try to understand this and correct it in a positive and positive way for comfortable joint interaction.

In the subsequent course of events, the doctor and the patient communicate, which can be divided into the following phases:

The contact formation phase is a short and very important phase, as it forms about $35 \%$ success with subsequent interaction with the patient. The examination process for the physician is formed from the first appearance of the patient: indicators of appearance, tone, voice, etc. In this case, the physician, like the patient in equal parts, assesses each other from the first moments of the treatment, which forms the basis of the future interaction and influence. In this phase, the main task is to create positive interaction between the physician and the patient, laying the groundwork that the physician wishes to help the patient and will take all possible measures [20].

Nonverbal communication, which manifests itself in facial expression, positive voice, and pronunciation, and open posture during conversation directed at the patient's eye, plays a large role in this task. In addition, the patient focuses on the appearance of the physician, as well as the state and cleanliness of his workplace, and assesses the doctor's cleanliness, the state of his clothes, and the degree of cleanliness. These indicators give a positive impression of the doctor, which is made up of precision, responsibility, and trust in both the person and the specialist of their work.

Next in line is the orientation phase, where the physician analyses the current state of the patient and the care to be provided on the basis of the patient's expectations and expectations. When seeing a doctor, the patient's condition can take different forms and carry various emotions, mostly negative, such as depression, indecision, and disorder. The physician shall throughout this phase reduce the emotional tension of the patient and make good contact with him necessary for further interaction [21].

During the entire orientation phase, you have to give the patient a voice, free from negative emotions and feelings. At the same time, if the patient is able to express his feelings and discuss them with the doctor, then another mechanism is activated, which by definition 3.Freud is called «catharsis» («purification», «liberation»)which is based on reducing emotional overload by describing your emotions verbally. In addition, during the description of the situation, the physician can assess the intelligence, character, level of development of the patient, as a result of which he will be able to find ways of communicating further.

At the moment when the doctor is listening to the patient, several options are put forward for the diagnosis, depending on the state of health, of the need for emergency treatment or the type of care, which are described in the subsequent stage of the argument.

During the argument phase, the moment of increased interaction begins, as a result of which the doctor collects additional necessary information to clarify and clarify the subtleties of the patient's state of health, check the proposed variants of diagnosis, and also the process of forming an opinion about the patient and choice of method of treatment. 
When a patient displays various negative emotions (apathy, aggression, etc.) in the course of conversation or interaction, the doctor needs to calm down, listen, tolerate, and show professional tact to the patient, try to persuade him and create positive feelings and try to enter into a relationship of trust, to persuade the patient.

From the above analysis, it can be concluded that no less important indicator of the formation of relations in a dyad doctor-patient is communication as a way of communicating information. It can also be assumed that one of the tasks of communication in the doctor-patient interaction is to perform not only an information function but also to overcome loneliness, to create a world in which other people are included besides the patient. On this basis, it can be said that communication is first and foremost interaction, the mere presence of another person already implies the transmission of information, and the functioning of the human being is constructed so, to make it easier or more difficult for another person to receive this information.

The next challenge for communicating in a given dyad is the patient doctor's call for help, in addition to communicating information and avoiding loneliness. The basis of the doctor-patient interaction as a psychological communicative phenomenon is the realization of an attempt of patients to call for help. The renowned American psychologist A. Megrobian believed that speech transmits only $7 \%$ of the information, $38 \%$ of the information is transmitted by paralinguistic elements, that is, the way the words are spoken, and $55 \%$ by the expression of the face. Postures, gestures, arm and leg movements are also very informative. Elements such as individual clothes, hairstyle, clothing accessories also carry significant information about this person. All the above are involved in communication processes [22], [23].

In his work, Erickson gave a great role to the non-verbal components of communication. Based on M.Erickson's research, investigators have come to the conclusion that regardless of specialization, doctors should be able to listen, see and feel more accurate and better than patients, moreover, they should consider non-verbal components of communication. Also, an essential point in the work of a doctor is the motivational component of interest in the activity and in the need to successfully carry out the assigned tasks, as a consequence, finding contact with the patient can be one of such tasks $[24,25]$.

\section{Results of the research}

The purpose of our research is to study the peculiarities of the doctor-patient personal compatibility in the process of healing interaction.

Research hypothesis: A patient visiting a doctor regularly chooses a specialist according to the personal qualities being the same with his or her ones. The Gissensk Personality Questionnaire was used as a research tool.

The Gissensk personality questionnaire (Gissensk test - GT) was developed in 1968 by a team of German scientists on the basis of the Psychosomatic Clinic of the University of Gissen as a diagnostic tool in the clinic, convenient for solving various questions of both individual and group diagnostics.

The adaptation of the questionnaire was carried out in accordance with the general rules of methodology creation and adaptation technology (Bodalev A.A., Stolin V.V., 1987) and included a translation of methodology, obtaining test standards, testing psychometric indicators (validity and reliability of the test).

Interpretation of basic scales: Each of the 6 basic scales has two poles:

1. The social acceptance scale at the lower pole implies a person's subjective idea of his or her social reputation, unattractiveness, others' disrespect, failure to achieve the objective, unpopularity. At the pole of high scores are those who are confident of their positive social 
image, attractiveness, popularity, ability to achieve a goal, respect, and appreciation of others. The main theme of this scale is the ability to interact successfully with the environment, a certain aspect of the social role.

2. The scale of dominance has at the lower pole ambition, an impatience, a desire to insist, on the other - obedience, compliance, patience. These complementary personality traits are identified with psychosocial protective patterns of behavior that contrast aggressiveness, impulsiveness, claims to primacy, complacency, inability to aggression, obedience, dependency.

3. Control scale (insufficient control - excessive control). The lower pole is characterized by sloppiness, volatility, inclination towards carefree behavior, reckless behavior, and an inability to dispose of money. Excessive control is characterized by meticulousness, diligence, truthfulness to fanaticism, lack of inclination towards frivolous, carefree behavior.

4. The scale includes questions about prevailing mood with two poles: hypomaniacal (it includes people who are rarely depressed, reflexive, critical, irritating, independent), depressive (often depressed, highly reflexive, timid, dependent, concealing frustration, very self-critical). The issues on the scale relate the prevailing mood to the basic direction of aggression for the individual.

5. The scale of extraversion and introversion. Those who show low performance on this scale describe themselves as gullible, social people with a strong need for love, frankness; At the other end of the scale, the subjects describe themselves as introverted, distrustful, detached from other people who seek to hide their need for love. This scale reflects the basic functions of social contacts and behavior, evolving from the primary trust or primary distrust (Erikson E. N., 1953).

The scale of social abilities - describes at one pole an active, communicative, relaxed competitive person with a rich imagination and the capacity for strong feelings. Social weakness, according to the authors, is characterized by lack of motivation, weak capacity for self-sacrifice, inability to long-term attachments, poor fantasy. The complexity of qualities reflected in this scale makes it possible to judge the degree of maturity of a person, where on the left pole a person is described as independent, active creative, confident in herself, capable of lasting and long-term interpersonal relations.

Participants in the study were: 3 medical workers with different specializations in higher medical education (1-41 years, 16 years of service; 2 - 35 years, 10 years of service; 3-42 years, 18 years of service), 45 patients aged $30-55$.

On the basis of the study, the following conclusions are drawn:

On the scale of success in interacting with the environment (social approval) doctors from groups 1 and 3 participating in the research are at a medium level. This fact makes it possible to assert that these doctors have an objective idea of their social reputation, attractiveness and popularity among others, as well as the ability to achieve their goals and respect among others.

A Group 2 doctor has a high score on this scale, indicating self-confidence in his abilities, self-identification as a person with a positive social reputation, and greater confidence in achieving the goals.

Of the middle-level patients on the scale of social interaction with the environment (social approval) 49 percent of the total number of patients (22 out of 45 ). It shows the similarities between doctors' and patients' opinions about themselves, their social reputation, attractiveness, etc. This number of patients is the highest percentage of the sample since $22 \%$ of patients have high values on this scale (10 out of 45 people) and low ones, which indicates subjectivity, unattractiveness, lack of respect for others and unpopularity - only $7 \%$ ( 3 out of 45 people). 
On the dominance scale, all psychotherapists have a middle level, indicating complementary personality traits. Depending on the situation, impulsiveness can be replaced by patience and the claim to primacy by accommodation and submission. Similarly, $69 \%$ of patients (31 out of 45 ) have an average level, while $9 \%$ (4 out of 45 ) have high rates. The remaining $22 \%$ (10 out of 45$)$ are at the lowest figures, indicating their impatience and willingness to insist.

On the control scale, all of the doctors involved in the study showed the average level, so that it can be argued that there is no frivolity and volatility, as well as that there is sufficient diligence in work, truthfulness and due diligence.

According to doctors, $78 \%$ of patients (35 out of 45 ) were examined. Their monitoring level is also in line with the average. A high level of control is held by $13 \%$ (6 out of 45 people), which means that they are mostly meticulous, diligent, truthful people with no propensity for frivolous behavior. Only 9\% (4 out of 45 people) have a low level of control, so they may be subtle, erratic, and frivolous.

On the mood scale, all doctors have similar indicators, which refer to the average level, which is an indication of their resistance to depression and self-criticism, as well as their resistance to excessive frustration and timidity. Among the patients, 71\% (32 out of 45) have a similar level. The low level is $13 \%$ ( 6 out of 45 people), indicating their depressed state, an inclination to reflex, concealment of annoyance and self-criticism. The rate is high at $16 \%$ (7 out of 45). People are rarely depressed, they are not critical, they do not conceal their irritation.

On the scale of extraversion - introversion mindsad, doctors in group 1 and group 2 have high scores, so they can be characterized as rather closed, sometimes detached and untrustworthy. A group 3 doctor has a middle level, which characterizes him as trustworthy, outgoing, partially open-minded. On this scale, the average is $73 \%$ (33 out of $45)$. High rates are found in $11 \%$ (5 out of 45). Low-level indicators are found in $16 \%$ ( 7 out of 45), which characterizes them as gullible, open and candid.

\section{Conclusions}

On the basis of the results obtained on the scale of social aptitudes, we have come to the conclusion that all doctors participating in the study have a medium level of social aptitude, which is indicative of competitiveness, activity and sociability. Among patients, 64\% (29 out of 45 ) have a similar rate. The low level is $36 \%$ (16 out of 45 people), which indicates that they are socially disadvantaged, have little capacity for commitment and are not capable of long-term attachments.

The compatibility analysis is carried out by the t-criterion Scientific, which is used to determine the statistical significance of differences in averages. In this case, the option for linked samples was used. The Gissenic Personality Questionnaire revealed that they are significant, i.e. they have an impact on the compatibility of the doctor and the patient, and the following indicators are statistically supported by the calculations: level of social acceptance and level of openness. At the same time, the level of control does not affect the compatibility between the doctor and the patient, which is also confirmed by the calculations made. Based on such indicators as mood, dominance and social ability, it can be concluded from the calculations that there is a predominant influence on the compatibility of doctor and patient.

The study shows that the largest number of patients who have been examined is the same as the physicians who participated in the study, which suggests a similarity between their personal characteristics and the predominant convergence of individual criteria. 
In our work, we looked for a relation between the personality of the doctor and the personality of the patients who visit him from time to time, as well as for similarities and differences between the doctor and the patient according to the parameters we choose.

The doctor-patient relationship is multifaceted. They require mutual respect, patience, mutual understanding, as well as many other factors that influence the positive interaction between doctor and patient. Compatibility is one of the most complex phenomenon of social and psychological science in general, and compatibility between doctor and patient plays a role in the success of the doctor's activities and the maintenance of the patient's health. A doctor is not only a professional, confident decision, encyclopedic knowledge but also the ability to speak to a patient. A doctor must have a «special sensitivity», the ability to feel compassion, as well as the ability to understand the patient and to comfort him if necessary. Trust is one of the most important factors in a good relationship and, in the case of a doctor-patient relationship, it is necessary, as often the patient has to trust the doctor with his life and health. Trust is defined as some interaction with certain requirements and criteria, and defined as the fulfillment of our expectations by the people on whom we depend, as well as trust has a close connection with honesty, decency, caring. All these are certain personal qualities that are investigated in this work.

In the course of a study on the personal compatibility of the physician and the patient, it can be concluded that the compatibility of the physician and the patient, and the existence of similar personal characteristics have a significant influence on the building of a literate relationship between them. The similarity of the personal characteristics of the doctor and the patient allows achieving the best result in their joint activity. In such a case, the physician will be able to fulfill his desire for help, and the patient will in turn respect the work of the physician.

Together, the patient's choice of doctor is influenced by the doctor's qualifications, his competence, and the patient's personal sympathy. The ability of a physician to empathize, that is, to empathize with the emotional state of the patient, plays an important role in the personal compatibility of the physician and the patient.

Thus, the hypothesis of the study was confirmed by the fact that the patient chooses a physician on the basis of his or her personal qualities.

\section{References}

1. L.E. Semenova, T.A. Serebryakova, Psychological well-being of psychology students with different personal resources of professionally significant characteristics, Bulletin of Minin University, 6(3) (2018)

2. Yu.E. Aleshina, Individual and family psychological counseling (2011)

3. R. Witch, Models of Moral Medicine in an Age of Revolutionary Change, Philosophical Issues, 3, 67-72 (1994)

4. A.A. Kirpichenko, Fundamentals of Medical Psychology and Communication, 174 (2004)

5. R. Konechny, Psychology in medicine, 405 (1983)

6. V.A. Bodrov, Psychological stress: development and overcoming, 528 (2006)

7. L.N. Vasilieva, Research of the communicative competence of a future doctor, 200 (2008)

8. K.K. Platonov, Entertaining Psychology, 300 (2011)

9. A.L. Sventsitsky, Psychology of Organizations Management, 222 (2009)

10. V. Boyko, A.G. Kovalev, V.N. Panferov, Socio-psychological climate of the team and personality, 207 (2013) 
11. N.N. Obozov, Three approaches to the study of psychological compatibility, Questions of Psychology, 6, 98-101 (1981)

12. M. Andreeva, N.N. Bogomolova, L.A. Petrovskaya, Foreign social psychology of the twentieth century, 286 (2012)

13. G.D. Babushkin, A.S. Bufius, Psychological compatibility and responsiveness in group activities, Psychopedagogy in law enforcement, 2, 40-42 (2006)

14. E.P. Ilyin, Psychology of communication and interpersonal relations, 576 (2011)

15. M.G. Kochurov, The influence of personal characteristics on interpersonal compatibility, 109 (2006)

16. E.D. Yastrebova, E.A. Pryakhina, A.A. Trifilova, N.V. Fomina, Experience in researching the relationship between a doctor and a patient by projective methods, International Student Scientific Bulletin, 5-1, 119 (2017)

17. E.J. Emanuel, Four models of the relationship between doctor and patient, [Electronic resource] http://jamanetwork.com/journals/jama/article-abstract/396718.

18. P.A. Clark, Medical practices 'sensitivity to patients' needs: Opportunities and practices for improvement, Journal of Ambulatory Care Management, 26(2), 110-123 (2003)

19. Z. Freud, Introduction to psychoanalysis, 437 (2015)

20. N.V. Fomina, T.E. Fedoseeva, I.V. Fomin, The attitude of doctors and patients to the treatment of the disease as a social psychological problem (on the example of the treatment of arterial hypertension), Bulletin of the Minin University, 2 (2016)

21. L.G. Pochebut, I.A. Meijis, Social psychology, 665 (2010)

22. G. Elwyn, A. Edwards, P. Kinnersley, R. Grol, Shared decision making and the concept of equipoise: the competences of involving patients in healthcare choices, British Journal of General Practice, 50, 892-899 (2000)

23. Defining the Patient-Physician Relationship for the 21st Century, 3rd Annual Disease Management Outcomes Summit, 2, (2003)

24. H.A. Murray, Explorations in personality, 516 (2008)

25. E.A. Zakharova, T.M. Sorokina, E.A. Yudina, Research actions in the structure of psychological readiness for the profession of a future doctor, Bulletin of Minin University, 7(3) (2019) 